\title{
Influence of mother tongue on dynamic handwriting features in primary school
}

\author{
P.D’Antrassi, F.Costa, E.Fornasa and A.Accardo \\ Department of Engineering and Architecture, University of Trieste, Via Valerio 10, Trieste, Italy
}

\begin{abstract}
Handwriting is an essential fine motor skill in school-aged children and plays a crucial role for educational development and autonomy of everyday life. To analyze handwriting movement an objective quantitative kinematic analysis can be performed by using digital tablets. Through this technology it was possible to identify a lot of parameters useful to characterize the handwriting process.

In order to study the influence of mother tongue on the dynamic of handwriting in primary school, we examined the writing response of 42 non-native speakers and compared their characteristics with those of 131 Italian mother tongue children. All children undertook one repetitive sequence of $l e$ and an Italian sentence written in two different ways: as accurately as and as fast as possible.

The results showed that the differences between native and non-native speakers were not significant in the repetitive sequence while a clear influence of mother tongue was present only in the third grade for the sentence tasks.
\end{abstract}

Keywords - Handwriting, mother tongue, kinematic analysis, motor skill.

\section{INTRODUCTION}

Handwriting is the result of a process in which linguistic, psychomotor and biomechanical factors interact with physical maturation, cognitive development and learning [1].

The study of fine motor movements is performed using characteristic parameters that measure precise kinematic features extracted from writing samples digitally recorded [2]. In particular, the factors concerning the basic elements of writing, such as strokes and components [3], proved to be very encouraging for hand motor performance quantification. A stroke is the basic element of writing movements, delimited by the points of minimal curvilinear velocity, while a component represents the segment between two successive pen lifts. The typical kinematic parameters related to strokes and components (like duration, length, mean and peak velocity) could also provide information on the level of automation and fluency achieved by a child [4].

To date, though many studies indicate changes in the values of some kinematic parameters depending on individual variables, such as age, gender, health status [5-7], only some authors examined the influence of mother tongue on the characteristics of handwriting [8,9].
Cheng et al. [8] investigated the class characteristics in English handwriting of three main racial groups: Chinese, Malay and Indian in Singapore. They studied several features such as letter designs, letter spacing, pen lifts and embellishments. Through a two-by-three chi-square analysis, they compared the frequency which each feature occurred in the groups, in order to identify the characteristic features peculiar to the individual racial group. Their results confirmed the impact of native language writing system on English handwriting.

Turnbull et al. [9] compared features between English and Polish handwriting performed by their respective mother tongue. Defining as class characteristic the features that occurred in $\geq 25 \%$ of the Polish handwriting, chi-square analyses was performed in order to identified the class characteristics that occurred significantly more in Polish compared to English handwriting. Their results supported the theory that class characteristics frequently stem from the taught writing system.

To our knowledge, no study examined the influence of Italian language in non-mother tongues' handwriting and in which stage of development possible alterations could occur in motor task performance. Thus, the aim of this paper is to investigate this influence underlining possible differences between Italian and non-Italian mother tongue subjects examining handwriting kinematic and static parameters in four different children pupils from $2^{\text {nd }}$ to $5^{\text {th }}$ grade of primary school. Each participant had to produce one repetitive cursive sequence, independent from linguistic aspects, and to copy a short meaningful sentence, which required adequate linguistic competences.

\section{MATERIALS AND METHODS}

\section{A. Study Population}

A sample of 173 students right-handed of different mother tongue, without handwriting problems or organic pathologies, was considered. Participants attended from primary school and their distribution along the four considered grades and the two groups (Italian mother tongue and nonnative speaker) is shown in Table 1.

Before starting the acquisition, written informed consent was obtained from the parents. 
Table 1 Number of students along the four considered grades divided in Italian $(\mathrm{GI} n)$ and non-Italian mother tongue (GNI $n)$ groups. $n$ represents the grade.

\begin{tabular}{ccc}
\hline grade & \#Italian mother tongue & \#non-native speaker \\
\hline $2^{\text {nd }}$ & $34(\mathrm{GI} 2)$ & $9(\mathrm{GNI} 2)$ \\
$3^{\text {rd }}$ & $23(\mathrm{GI} 3)$ & $12(\mathrm{GNI})$ \\
$4^{\text {th }}$ & $32(\mathrm{GI} 4)$ & $13(\mathrm{GNI})$ \\
$5^{\text {th }}$ & $42(\mathrm{GI} 5)$ & $8(\mathrm{GNI})$ \\
\hline
\end{tabular}

\section{B. Tasks}

In order to study possible parameter dependence on linguistic competences acquired by both Italian and non-native speakers, all children performed a series of three tasks:

LE task Test partially independent from linguistic aspects; required pupils to write, for a minute, as fast as possible, a cursive sequence of $l e$;

$A$ and $F$ tasks Tests in which adequate linguistic competences are required; asked pupils to copy in cursive, as accurately as possible (A) and as fast as possible (F), the Italian sentence: L'elefante vide benissimo quel topo che rubava qualche pezzo di formaggio (meaning The elephant clearly saw that mouse stealing some pieces of cheese). This sentence was constructed in order to contain all the letters of the Italian alphabet.

\section{Data Acquisition and Analysis}

Pen displacement was sampled at $200 \mathrm{~Hz}$, both horizontally and vertically by using a commercial digitizing tablet with a spatial resolution of $5 \mu \mathrm{m}$ (Wacom, Inc., Vancouver, WA, Model Intuos3). An ink pen and a sheet of a lined paper, appropriate to the grade attended, was used, in order to reproduce a normal pen and paper context.

A proprietary MATLAB script [10] was used to perform the analysis. To estimate the curvilinear position and velocity curves, the horizontal and vertical pen positions were separately filtered by means of a $2^{\text {nd }}$ order low-pass Butterworth filter $\left(f_{c}=10 H z\right)$ and suitably combined. Components were identified as the written tracts between two consecutive pen lifts and strokes as the segments between two successive points of minimal curvilinear velocity, as claimed by the bell-shaped velocity profile theory [11].

To study possible changes of handwriting kinematic characteristics the mean value and the standard deviation of some parameters (Table 2), were calculated for each task in all the children. The results were averaged across pupils of the same mother tongue and grade. For each task and grade $(n)$, the significance of the difference of each parameter between the two GI $n$ and GNI $n$ groups, was evaluated by means of the Wilcoxon rank sum test.
Table 2 List of kinematic and static parameters considered

\begin{tabular}{clc}
\hline ID & \multicolumn{1}{c}{ Parameter } & Unit \\
\hline TL & Total length of the task & $\mathrm{mm}$ \\
TD & Total duration of the task & $\mathrm{ms}$ \\
PL & Pen lift duration & $\mathrm{ms}$ \\
SN & Number of strokes & $\#$ \\
SL & Mean length of each stroke & $\mathrm{mm}$ \\
SD & Mean duration of each stroke & $\mathrm{ms}$ \\
TV & Curvilinear velocity of the task & $\mathrm{mm} \cdot \mathrm{s}^{-1}$ \\
SV & Mean curvilinear velocity of each stroke & $\mathrm{mm} \cdot \mathrm{s}^{-1}$ \\
SN/TD Number of strokes / s & $\mathrm{s}^{-1}$ \\
SN/LN & Number of strokes / number of letters & $\#$ \\
LN/TL Number of letters / cm & $\mathrm{cm}^{-1}$ \\
\hline
\end{tabular}

\section{Results}

Significant differences $(\mathrm{p} \leq 0.05)$ between Italian mother tongue and non-native speakers were observed only in the third grade on a few parameters, especially during the sentence tasks (A and F tasks). These parameters were: TL; PL; SN; SD; SN/TD; SN/LN; LN/TL (Table 3) and TD (Figure 1). For TV, SV and SL no significant difference was observed.

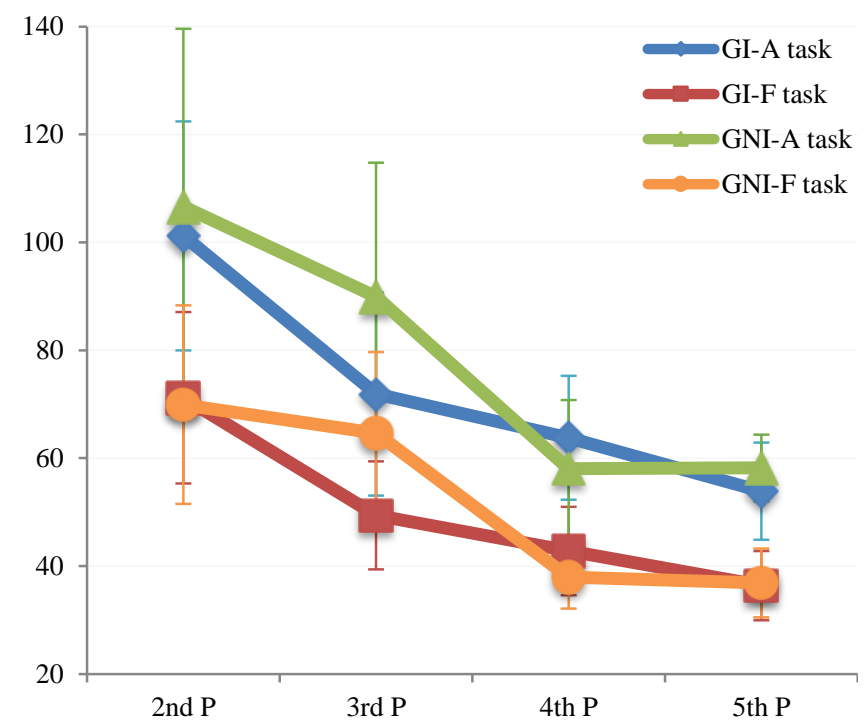

Figure 1 Total duration of the track, TD (in ms), performed by Italian (GI) and non-Italian mother tongue (GNI) groups along the grades for $\mathrm{A}$ and $\mathrm{F}$ tasks

Figure 1 shows the behavior of the Total Duration of the track along the grades: pupils reduced their time to perform the sentence task with schooling, irrespective of their group except for $3^{\text {rd }}$ grade, in which the two groups showed significant differences both in $\mathrm{A}(\mathrm{p}<0.04)$ and in $\mathrm{F}(\mathrm{p}<0.004)$ tasks. 
VI Congreso Latinoamericano de Ingeniería Biomédica

VI Cogresso Latino-americano de Engenharia Biomédica

VI Latin American Conference in Biomedical Engineering

Table 3 Parameters showing significant differences between Italian mother tongue and the others calculated for LE, A and F tasks.

\begin{tabular}{|c|c|c|c|c|c|c|c|c|c|}
\hline & \multicolumn{9}{|c|}{ TL - Total length of track [mm] } \\
\hline & \multicolumn{3}{|c|}{ Italian mother tongue } & \multicolumn{3}{|c|}{ No Italian mother tongue } & \multicolumn{3}{|c|}{ p-value } \\
\hline & LE task & A task & F task & LE task & A task & F task & LE task & A task & F task \\
\hline $2^{\text {nd }} \mathrm{P}$ & $133,2 \pm 26,9$ & $131,2 \pm 27,3$ & $135,3 \pm 30$ & $150,4 \pm 28$ & $129 \pm 15,2$ & $132,8 \pm 24$ & n.s. & n.s. & n.s. \\
\hline $3^{\text {rd }} \mathrm{P}$ & $145,4 \pm 30,5$ & $95,1 \pm 11,2$ & $103,7 \pm 19,2$ & $152,3 \pm 62,4$ & $122,4 \pm 37,2$ & $129,1 \pm 34,9$ & n.s. & 0,002 & 0,013 \\
\hline $4^{\text {th }} \mathrm{P}$ & $131,3 \pm 22,4$ & $88,9 \pm 20,2$ & $95,9 \pm 19,3$ & $132,4 \pm 21,5$ & $83,7 \pm 11,4$ & $86 \pm 12,8$ & n.s. & n.s. & n.s. \\
\hline \multirow[t]{4}{*}{$5^{\text {th }} \mathrm{P}$} & $133,1 \pm 28,8$ & $80,3 \pm 14$ & $85,2 \pm 14,6$ & $141,8 \pm 22,9$ & $79,8 \pm 11,5$ & $82,9 \pm 11,9$ & n.s. & n.s. & n.s. \\
\hline & \multicolumn{9}{|c|}{ PL - Pen lift duration [ms] } \\
\hline & \multicolumn{3}{|c|}{ Italian mother tongue } & \multicolumn{3}{|c|}{ No Italian mother tongue } & \multicolumn{3}{|c|}{ p-value } \\
\hline & LE task & A task & $\mathrm{F}$ task & LE task & A task & $\mathrm{F}$ task & LE task & A task & F task \\
\hline $2^{\text {nd }} \mathrm{P}$ & $4,4 \pm 3,3$ & $35,5 \pm 10,9$ & $25,7 \pm 9,4$ & $5,9 \pm 3,4$ & $39,1 \pm 15,5$ & $23,9 \pm 7,8$ & n.s. & n.s. & n.s. \\
\hline $3^{\text {rd }} \mathrm{P}$ & $6,9 \pm 5$ & $27,8 \pm 10$ & $17,5 \pm 5,8$ & $5,8 \pm 3,7$ & $31,9 \pm 9,1$ & $23,2 \pm 7$ & n.s. & n.s. & 0,019 \\
\hline $4^{\text {th }} \mathrm{P}$ & $5,8 \pm 3,5$ & $23,3 \pm 6,7$ & $14 \pm 5,1$ & $3,9 \pm 2,3$ & $19,2 \pm 4,7$ & $10,7 \pm 2,9$ & n.s. & 0,055 & 0,022 \\
\hline \multirow[t]{4}{*}{$5^{\text {th }} \mathrm{P}$} & $4,1 \pm 2,6$ & $19,1 \pm 5,8$ & $10,9 \pm 3,9$ & $4,5 \pm 2,8$ & $20,3 \pm 4,4$ & $10,6 \pm 3,7$ & n.s. & n.s. & n.s. \\
\hline & \multicolumn{9}{|c|}{ SN - \#Stroke } \\
\hline & \multicolumn{3}{|c|}{ Italian mother tongue } & \multicolumn{3}{|c|}{ No Italian mother tongue } & \multicolumn{3}{|c|}{ p-value } \\
\hline & LE task & A task & F task & LE task & A task & F task & LE task & A task & F task \\
\hline $2^{\text {nd }} \mathrm{P}$ & $273,6 \pm 29$ & $402,4 \pm 96,1$ & $299,4 \pm 53,1$ & $259,3 \pm 17,2$ & $416,3 \pm 114,9$ & $302,6 \pm 55,4$ & n.s. & n.s. & n.s. \\
\hline $3^{\text {rd }} \mathrm{P}$ & $260,9 \pm 28,4$ & $294,9 \pm 60$ & $245,5 \pm 35,5$ & $284,7 \pm 37,5$ & $363,9 \pm 92$ & $290,3 \pm 39,3$ & n.s. & 0,014 & 0,004 \\
\hline $4^{\text {th }} \mathrm{P}$ & $275,8 \pm 25,5$ & $270,6 \pm 32$ & $223,3 \pm 31,9$ & $283,3 \pm 6,4$ & $282,7 \pm 57,7$ & $217,8 \pm 25,2$ & n.s. & n.s. & n.s. \\
\hline \multirow[t]{4}{*}{$5^{\text {th }} \mathrm{P}$} & $282,4 \pm 16,6$ & $247,6 \pm 30,1$ & $204,9 \pm 26,6$ & $279,1 \pm 11,1$ & $262,9 \pm 19,5$ & $202,3 \pm 17,7$ & n.s. & n.s. & n.s. \\
\hline & \multicolumn{9}{|c|}{ SD - Mean Stroke Duration [ms] } \\
\hline & \multicolumn{3}{|c|}{ Italian mother tongue } & \multicolumn{3}{|c|}{ No Italian mother tongue } & \multicolumn{3}{|c|}{ p-value } \\
\hline & LE task & A task & F task & LE task & A task & F task & LE task & A task & F task \\
\hline $2^{\text {nd }} \mathrm{P}$ & $186 \pm 21,4$ & $157,5 \pm 15$ & $148,7 \pm 13,8$ & $195,9 \pm 15,5$ & $154,1 \pm 12,3$ & $150,3 \pm 10,2$ & n.s. & n.s. & n.s. \\
\hline $3^{\text {rd }} \mathrm{P}$ & $196,8 \pm 17,5$ & $145,3 \pm 14,4$ & $128 \pm 10$ & $173,8 \pm 25$ & $152,5 \pm 19,3$ & $138,4 \pm 19,2$ & 0,004 & n.s. & 0,051 \\
\hline $4^{\text {th }} \mathrm{P}$ & $192,6 \pm 20,5$ & $148,6 \pm 15,5$ & $128,8 \pm 10,7$ & $191,5 \pm 18,3$ & $137 \pm 10,9$ & $123,9 \pm 11,7$ & n.s. & 0,020 & n.s. \\
\hline \multirow[t]{4}{*}{$5^{\text {th }} \mathrm{P}$} & $192,7 \pm 15,5$ & $137,4 \pm 13$ & $123,5 \pm 9,8$ & $193,5 \pm 6,2$ & $146,4 \pm 17,9$ & $127,3 \pm 10,7$ & n.s. & n.s. & n.s. \\
\hline & & & & SN/TD - \# & $\mathrm{ke} / \mathrm{s}\left[\mathrm{s}^{-1}\right]$ & & & & \\
\hline & & ian mother ton & & & alian mother to & & & p-value & \\
\hline & LE task & A task & $\mathrm{F}$ task & LE task & A task & F task & LE task & A task & F task \\
\hline $2^{\text {nd }} \mathrm{P}$ & $4,56 \pm 0,48$ & $3,99 \pm 0,56$ & $4,28 \pm 0,6$ & $4,36 \pm 0,29$ & $3,97 \pm 0,52$ & $4,42 \pm 0,56$ & n.s. & n.s. & n.s. \\
\hline $3^{\text {rd }} \mathrm{P}$ & $4,35 \pm 0,47$ & $4,2 \pm 0,63$ & $5,03 \pm 0,54$ & $4,74 \pm 0,62$ & $4,1 \pm 0,6$ & $4,61 \pm 0,63$ & n.s. & n.s. & 0,036 \\
\hline $4^{\text {th }} \mathrm{P}$ & $4,6 \pm 0,42$ & $4,31 \pm 0,55$ & $5,29 \pm 0,66$ & $4,72 \pm 0,44$ & $4,91 \pm 0,56$ & $5,77 \pm 0,5$ & n.s. & 0,002 & 0,021 \\
\hline $5^{\text {th }} \mathrm{P}$ & $4,72 \pm 0,27$ & $4,66 \pm 0,64$ & $5,68 \pm 0,56$ & $4,65 \pm 0,18$ & $4,57 \pm 0,64$ & $5,58 \pm 0,77$ & n.s. & n.s. & n.s. \\
\hline & & & & $\mathrm{SN} / \mathrm{LN}-\#$ & e/Letter & & & & \\
\hline & & ian mother ton & & & alian mother to & & & $\mathrm{p}$-value & \\
\hline & LE task & A task & $\mathrm{F}$ task & LE task & A task & $\mathrm{F}$ task & LE task & A task & F task \\
\hline $2^{\text {nd }} P$ & $4,62 \pm 1,15$ & $6,39 \pm 1,53$ & $4,75 \pm 0,84$ & $3,97 \pm 0,72$ & $6,69 \pm 1,83$ & $4,9 \pm 0,78$ & n.s. & n.s. & n.s. \\
\hline $3^{\text {rd }} \mathrm{P}$ & $3,36 \pm 0,52$ & $4,68 \pm 0,95$ & $3,9 \pm 0,56$ & $4,49 \pm 0,99$ & $5,83 \pm 1,48$ & $4,64 \pm 0,6$ & 0,002 & 0,014 & 0,003 \\
\hline $4^{\text {th }} \mathrm{P}$ & $3,36 \pm 0,66$ & $4,3 \pm 0,51$ & $3,54 \pm 0,51$ & $3,22 \pm 0,54$ & $4,49 \pm 0,92$ & $3,44 \pm 0,39$ & n.s. & n.s. & n.s. \\
\hline $5^{\text {th }} \mathrm{P}$ & $3,25 \pm 0,58$ & $3,93 \pm 0,48$ & $3,25 \pm 0,42$ & $3,2 \pm 0,28$ & $4,17 \pm 0,31$ & $3,21 \pm 0,28$ & n.s. & n.s. & n.s. \\
\hline & & & & $\mathrm{LM} / \mathrm{TL}-\# \mathrm{~L}$ & $/ \mathrm{cm}\left[\mathrm{cm}^{-1}\right]$ & & & & \\
\hline & & ian mother ton & & & alian mother to & & & p-value & \\
\hline & LE task & A task & $\mathrm{F}$ task & LE task & A task & $\mathrm{F}$ task & LE task & A task & F task \\
\hline $2^{\text {nd }} P$ & $0,46 \pm 0,04$ & $0,5 \pm 0,11$ & $0,48 \pm 0,09$ & $0,45 \pm 0,03$ & $0,49 \pm 0,06$ & $0,48 \pm 0,08$ & n.s. & n.s. & n.s. \\
\hline $3^{\text {rd }} \mathrm{P}$ & $0,55 \pm 0,06$ & $0,67 \pm 0,08$ & $0,63 \pm 0,1$ & $0,46 \pm 0,09$ & $0,54 \pm 0,12$ & $0,51 \pm 0,11$ & 0,004 & 0,002 & 0,008 \\
\hline $4^{\text {th }} \mathrm{P}$ & $0,65 \pm 0,09$ & $0,75 \pm 0,17$ & $0,68 \pm 0,14$ & $0,68 \pm 0,06$ & $0,76 \pm 0,09$ & $0,75 \pm 0,1$ & n.s. & n.s. & n.s. \\
\hline $5^{\text {th }} \mathrm{P}$ & $0,68 \pm 0,09$ & $0,81 \pm 0,14$ & $0,76 \pm 0,13$ & $0,63 \pm 0,09$ & $0,81 \pm 0,12$ & $0,78 \pm 0,12$ & n.s. & n.s. & n.s. \\
\hline
\end{tabular}

\section{Discussion}

In LE task, in which language skills required are minimal and a major motor effort is necessary, the differences between Italian mother tongue and non-native pupils generally are not significant.

On the other hand, in the $\mathrm{A}$ and $\mathrm{F}$ tasks mother tongue in- fluences is clear only in the third grade. In all the other primary school grades, no significant differences are observed.

From the results of current study we can observe than during the first year of learning cursive, only a slight influence of mother tongue is present probably because in the pupils of the second grade the graphical description of letters prevails on their linguistic representation. On the contrary when students become more aware of what the sen- 
tence means, in third grade, significant differences between native and non-native speakers exist and affect many handwriting characteristics like, for example, the number of strokes per letter that is greater in non-native pupils. In the successive grades (fourth and fifth), this linguistic gap between native and non-native speakers is recovered, in terms of dynamic handwriting features, by training and practice language.

\section{v. Conclusions}

During the primary school, pupils writing in a language different from the native one, highlights significant differences only in third grade. That may have something to do with the acquisition of linguistic skills, other than handwriting automation. After the second grade, in fact, writing stops being a mechanical process and becomes a cognitive product. Adequate linguistic skills must therefore be acquired by non-native speakers before to write as native ones.

\section{ACKNOWLEDGMENT}

Work partially supported by the University of Trieste, Master in Clinical Engineering. The authors thank the Dept. of Children Neuropsychiatry of Institute for Maternal and Child Health - IRCCS Burlo Garofolo of Trieste for the valuable collaboration in handwriting data collection.

\section{CONFLICT OF INTEREST}

The authors declare that they have no conflict of interest.

\section{REFERENCES}

1. Accardo A., Genna M., Borean Michela (2013) Development, maturation and learning influence on handwriting kinematics. Human Movement Science Vol. 32, pp. 136-146
2. Erasmus LP, Sarno S, Albrecht H et al (2001) Measurement of ataxic symptoms with a graphic tablet: standard values in controls and validity in Multiple Sclerosis patients. J.Neursci. Methods 108:25-37

3. Van Galen GP, Weber JF (1998) On-line size control in handwriting demonstrates the continuous nature of motor programs. Acta Psychol. (Amst) 100:195-216

4. Rosenblum S, Chevion D, Weiss PL (2006) Using data visualization and signal processing to characterize the handwriting process. Pediatric Rehabil 4:404-17

5. Coksevim B, Caksen H (2005) Evaluation of exercise performance in healthy Turkish adolescents. Int $\mathbf{J}$ Neurosci 115(7):1033-9

6. Davies PL, Rose JD (2000) Motor skills of typically developing adolescents: awkwardness or improvement? Phys Occup Ther Pediatr 20(1):19-42

7. Genna M., Accardo A. (2011) Gender and Age Influence in handwriting Performance in Children and Adolescents, IFMBE Proc. Vol. 37, $5^{\text {th }}$ European IFMBE Conference, pp. 141-144

8. Cheng N, Lee GK, Yap BS, Lee LT, Tan SK, Tan KP (2005) Investigation of class characteristics in English handwriting of the three main racial groups: Chinese, Malay and Indian in Singapore. J Forensic Sci 50(1): 1-8

9. Turnbull SJ, Jones AE, Allen M (2010) Identification of the class characteristics in the handwriting of Polish people writing in English. Journal of Forensic Sciences, Vol. 55, No. 5

10. Accardo A, Chiap A, Borean M et al (2007) A device for quantitative kinematic analysis of children's handwriting movements. IFMBE Proc. Vol.16, MEDICON 2007, Lubljiana, Slovenija, 2007, pp 445-448

11. Djioua M, Plamondon R (2009) A new algorithm and system for the characterization of handwriting strokes with delta-lognormal parameters. IEEE Trans Pattern Anal Mach Intell 31(11):20602072

Address of the corresponding author:

$\begin{array}{ll}\text { Author: } & \text { D'Antrassi Pierluigi } \\ \text { Institute: } & \begin{array}{l}\text { Department of Engineering and Architecture, } \\ \text { University of Trieste }\end{array} \\ \text { Street: } & \text { Via Valerio, } 10 \\ \text { City: } & \text { Trieste (TS) } \\ \text { Country: } & \text { Italy } \\ \text { Email: } & \text { pierluigi.d'antrassi@phd.units.it: }\end{array}$

